\title{
A Strategy for In-Silico Prediction of Skin Absorption in Man
}

Dominik Selzer ${ }^{1}$, Dirk Neumann², Heike Neumann${ }^{1}$, Karl-Heinz Kostka ${ }^{3}$, ClausMichael Lehr ${ }^{1,4}$, Ulrich F. Schaefer ${ }^{1 *}$

1 Biopharmaceutics and Pharmaceutical Technology, Saarland University, D-66123 Saarbruecken, Germany

2 Scientific Consilience, Saarland University, D-66123 Saarbruecken, Germany

3 Dept. of Plastic and Hand Surgery, Caritaskrankenhaus, D-66822 Lebach, Germany

4 Dept. of Drug Delivery (DDEL), Helmholtz Institute for Pharmaceutical Research Saarland (HIPS), Helmholtz Center for Infection Research (HZI), Saarland University, D-66123 Saarbruecken, Germany

* Author, to whom correspondence should be directed

telephone number: + 49/681/302 2019

fax number: + 49 / 681 / 3024677

e-mail address: ufs@mx.uni-saarland.de

\section{ABSTRACT}

For some time, in-silico models to address substance transport into and through the skin are gaining more and more importance in different fields of science and industry. In particular, the mathematical prediction of in-vivo skin absorption is of great interest to overcome ethical and economical issues. The presented work outlines a strategy to address this problem and in particular, investigates in-vitro and in-vivo skin penetration experiments of the model compound flufenamic acid solved in an ointment by means of a mathematical model. Experimental stratum corneum concentration-depth profiles (SC-CDP) for various time intervals using two different in-vitro systems (Franz diffusion cell, Saarbruecken penetration model) were examined and simulated with the help of a highly optimized three compartment numerical diffusion model and compared to findings of SC-CDPs of the in-vivo scenario. Fitted model input parameters (diffusion coefficient and partition coefficient with respect to the stratum corneum) for the in-vitro infinite dose case could be used 
to predict in-use conditions in-vitro. Despite apparent differences in calculated partition coefficients between in-vivo and in-vitro studies, prediction of in-vivo scenarios from input parameters calculated from the in-vitro case yielded reasonable results.

\section{KEY WORDS}

- Skin concentration-depth profiles

- In-vivo studies

- In-vitro studies

- Diffusion model

- Semisolid drug preparations

\section{RESEARCH HIGHLIGHTS}

- Strategy for in-silico prediction of in-use in-vivo skin penetration

- Numerical modeling of stratum corneum concentration-depth profiles over time

- Concept and model validation with experimental tape-stripping data

- In-vitro infinite dose model parameters can be use to predict in-use conditions

- In-vitro model input parameters are suitable to predict in-vivo scenarios 


\section{Abbreviations:}

$\begin{array}{ll}\text { SC } & \text { Stratum corneum } \\ \text { SB-M } & \text { Saarbruecken penetration model } \\ \text { FD-C } & \text { Franz diffusion cell } \\ \text { FFA } & \text { Flufenamic acid } \\ Q & \text { Released amount per area } \\ t & \text { Time } \\ C_{0} & \text { Initial concentration at time } \mathrm{t}=0 \\ k_{p} & \text { Permeability } \\ D & \text { Diffusion coefficient } \\ K & \text { Partition coefficient } \\ h & \text { Height of the membrane or barrier } \\ c & \text { Concentration } \\ \text { DSL } & \text { Deeper skin layers (viable epidermis and parts of the dermis) } \\ \text { RSE } & \text { Residual standard error }\end{array}$

\section{INTRODUCTION}

The importance of substance transport into and through the skin has grown consistently in different fields of science and industry over the past decade. Not only pharmaceutical applications for the local or systemic administration of drugs or applications in the cosmetic industries rely on careful investigations of the underlying transport kinetics, especially regulation authorities such as REACH (Regulation, Evaluation, Authorization and Restriction of Chemicals) (1) request information about characteristics of potential harmful xenobiotic exposure to the human skin $(2,3)$.

In-vivo experiments in the field of skin absorption are considered the gold standard but are difficult to perform and evaluate due to the complexity of processes involved 
and obviously face ethical concerns $(4,5)$. Hence, experiments with in-vitro test systems are used frequently to provide this information (6-8). In the past, it has been shown repeatedly that these test systems are capable of mimicking the in-vivo situation reasonably (9-11).

Many investigations in dermal research are performed under infinite dose conditions. These studies are done by applying a sufficiently large amount of the test formulation to the surface of the skin, so that neither the drug nor any of the vehicular components are appreciably reduced during the course of the experiment. The advantage of this procedure of the application of the vehicle is very reproducible and quasi steady-state conditions may be reached in the stratum corneum (SC) (12). Effects caused by rubbing are also avoided. On the other hand, this procedure does not mimic applications as they are done in practice, where typically small quantity of drug preparation (finite dose) is applied on a relatively large area of the skin by massaging.

Today, considerable information about drug distribution within the skin, following infinite dosing, is available. In contrast, the data for finite dosing is rather limited (13), Data for semisolid drug preparation is also less common than permeation data .

Based on available data (14) it was decided to carry out a study gathering input parameters (SC diffusivity and partition coefficient) with respect to a mathematical model based on experimental results of two different in-vitro test systems, the Saarbruecken penetration model (SB-M) and the Franz diffusion cell (FD-C), using the tape stripping technique (15). With the help of an in-silico model these input parameters were used to predict the situation in-vivo. Experimental data was examined under infinite dose and finite dose conditions for the aforementioned test systems and in-vivo studies. Finite dosing was carried out on the basis of different guidance documents, which state that finite dosing is given when the amount of 
ointment applied to the skin is between $1-10 \mathrm{mg} / \mathrm{cm}^{2}(16-18)$. Consequently, infinite dose conditions were implemented by application of more than $10 \mathrm{mg} / \mathrm{cm}^{2}$ of formulation. These procedures were performed with a semisolid drug preparation. Mathematical models predicting skin absorption have, for some time, been considered as alternatives to experimental investigations by different regulatory agencies (19-21). For complex mechanistic models it could be shown that in-vitro infinite and finite dose concentration-depth profiles could be predicted reasonably but require a complex set of input parameters (22-24). In contrast, in the current work a sparse parameter one-dimensional diffusion model was used to not only investigate and predict the in-vitro situation but also to predict the in-vivo situation based on input parameters derived from an in-vitro based model. For this purpose, the underlying diffusion equation was solved numerically. First of all, the model was fitted to in-vitro concentration-depth profiles to obtain information about diffusivity and partition coefficient with respect to the SC. Subsequently, in-vitro infinite dose fitting results were used to predict the in-vitro finite dose scenario as conducted successfully before for aqueous formulations $(22,25)$. Finally, the model was used to predict invivo concentration-depth profiles for the finite and infinite dose case

A positive outcome of the presented study should not only give a better hint on the theory of the drug's diffusion into the SC but most likely be a starting point to reduce experimental work in-vitro and in-vivo. It should therefore reduce costs and time required for the development of semisolid drug formulations and other formulations for dermal application.

\section{Materials and Methods}

The following materials and equipment were used: Flufenamic acid (Kali-Chemie Pharma, Hannover, D); wool alcohols ointment and Multifilm kristall-klar (Beiersdorf, 
Hamburg, D); Ringer solution, Mcllvaine citric acid-phosphate buffer pH 2.2, $\mathrm{NaOH}$ (Merck, Darmstadt, D); Plastibase ${ }^{\circledast}$ (Heyden GmbH, Muenchen, D); methanol (Baker, Deventer, NL); Franz diffusion cells type 4G-01-00-20-15, area $=3.142 \mathrm{~cm}^{2}$, acceptor volume $=15 \mathrm{ml}($ Perme Gear, Riegelsville, PA, USA) teflon filter Minisart - pore size $0.2 \mu \mathrm{m}$ (Sartorius, Goettingen, D); isocratic HPLC consisting of a 655 A 40 autosampler, L 4250 detector, L 6220 pump, $6000 \mathrm{~K}$ data interface and $5 \mu \mathrm{m}$ LiChrospher $^{\circledR} 100$ / RP-18 column / 12.5 cm x 4 mm (Merck-Hitachi, Darmstadt, D); Dialysis membrane, cut off 10,000 (Dianorm GmbH, Munich, Germany); Cellulose membrane, cut off 10,000 (Medicell International LTD, London, GB).

The non-steroidal drug flufenamic acid (FFA), solved in a concentration of $0.9 \%$ in wool alcohols ointment (German Pharmacopoeia 1999), was used as drug preparation under infinite and finite dose conditions. Prior to the application, the drug preparation was stored at $32^{\circ} \mathrm{C}$ for one week to allow complete dissolution of the drug in the ointment base. The absence of drug particles was checked by light microscopic investigations.

\section{Skin samples for in-vitro experiments}

Skin samples were taken from Caucasian female donors undergoing abdominal surgery with the approval of the ethic committee of the Caritas-Hospital Lebach, Germany. Immediately after excision the subcutaneous fatty tissue was removed using a scalpel. The skin was cut into $10 \times 10 \mathrm{~cm}$ pieces, wrapped in aluminium foil and stored in polyethylene bags at $-26^{\circ} \mathrm{C}$ until use. The maximum storage time was three months.

\section{Saarbruecken penetration model (SB-M) experiments}


Details of the experiments are given in (10). Briefly, for infinite dosing an ointment layer of at least $2 \mathrm{~mm}$ was applied whereas for finite dosing 4-6 mg ointment per $\mathrm{cm}^{2}$ was evenly spread on the skin surface. All experiments were carried out at a skin surface temperature of $32 \pm 1^{\circ} \mathrm{C}$ for different time intervals $(0.5,1,3$ and 6 hours) at occlusive conditions.

\section{Franz diffusion cell (FD-C) experiments}

Details of the experiments are given in (10). In short, application of the ointment was conducted similar to the procedure described previously (SB-M). As receptor fluid Soerensen phosphate buffer $\mathrm{pH} 7.4$ was used and mixed with a magnetic stirring bar at $500 \mathrm{rpm}$. To allow equilibration the skin specimen was pre-equilibrated for 30 minutes prior to the application of the formulation. All experiments were carried out as described in the previous section (SB-M).

\section{Horizontal segmentation of the stratum corneum (SC)}

To compare the results of both models, the skin was always treated in exactly the same manner at the end of all experiments (10). First, the remaining ointment was removed by wiping the skin with cotton. Second, the skin was successively stripped with 20 pieces of adhesive tape. In a standardised procedure (pressure: $2 \mathrm{~kg}$ for 10 sec), tapes were removed rapidly and combined in 6 pools of $1,1,3,4,5$ and 6 strips for analytical purposes. Due to this procedure, each of the removed cell layers had nearly the same thickness, which had been shown in previous studies in different laboratories (26-28). The first tape strip was always discarded because of potential contamination. 


\section{In-vivo experiments}

6 human volunteers ( 3 male, 3 female), aged 23 - 29 years, from whom informed consent was obtained, participated in the study (10). They were in good health and had no history of any dermatological disease.

A template of Fixomull ${ }^{\circledR}$ with 4 holes was fixed on the volar left and right forearm of each volunteer. Each hole released an area of $15 \mathrm{~mm}$ in diameter and represented one experimental area. The drug preparation was applied according to the in-vitro experiments. The administration areas were not covered during the incubation time $(0.25,0.5,1$ and $3 \mathrm{~h})$, but the volunteers were asked to reduce their movements to avoid any loss of drug preparation.

The tape-stripping procedure (see the previous section) was performed by exerting the pressure just with the forefinger. To increase reproducibility, the procedure was carried out by the same person according to the AAPS/FDA Workshop report (29) and the Guidance for Industry (30).

\section{In-vitro release experiments}

The release of FFA from the ointment was examined for different concentrations $(0.225 \%, 0.45 \%, 0.675 \%$ and $0.9 \%)$ using an infinite dose setup (approximately $0.3 \mathrm{~g}$ of applied formulation). For that reason, by means of a FD-C setup, released mass over time across a dialysis membrane (0-6 h, 5 time points) and cellulose membrane (0-53 h, 11-18 time points) was determined, respectively.

\section{Assay method and HPLC-procedure}

For a detailed description of the extraction procedure and the HPLC analysis [detection limit $20 \mathrm{ng} / \mathrm{ml}$ ] see Wagner et al. (10). The linearity of the HPLC method 
was checked over a quantification range from 50 to $10,000 \mathrm{ng} / \mathrm{ml}$, providing a good linearity between the area of the UV-absorption peak and the respective concentration with $r=0.999$ and an accuracy of $97.8 \pm 3.8 \%$.

\section{Determination of diffusivity of FFA in the ointment}

As stated before, the release of FFA for different concentrations $(0.225 \%, 0.45 \%$, $0.675 \%$ and $0.9 \%$ ) was examined using a dialysis membrane and a cellulose membrane. To estimate diffusivity $D$ of FFA inside the vehicle the amount released per area $Q$ over time $t$ for initial concentration $C_{0}$ (assuming a uniform distribution of FFA within the ointment at $t=0$ ) was fitted for every single experiment using a nonlinear least squares approach (Eq. 1) (31).

$Q=2 C_{0}\left(\frac{D t}{\pi}\right)^{\frac{1}{2}}$

Eq. 1

Since equation 1 only holds true for a loss of initial concentration of approximately up to $30 \%$ over time, the general solution (Eq. 2) with thickness of applied ointment layer $h \quad(h=2 \mathrm{~mm})$ was applied to analyse the impact of a potential loss of initial concentration and ensure valid results with respect to the diffusion coefficient (31).

$Q=h C_{0}\left[1-\frac{8}{\pi^{2}} \sum_{m=0}^{\infty} \frac{1}{(2 m+1)^{2}} \exp \left(-\frac{D(2 m+1)^{2} \pi^{2} t}{4 h^{2}}\right)\right]$

Eq. 2

Here, the underlying infinite sum was calculated until maximum possible accuracy was reached (comparison to the machine epsilon) (24).

\section{Diffusion model}


The diffusion of FFA from the ointment through the SC was simulated using a onedimensional diffusion model. Three compartments (vehicle, SC, deeper skin layers) were implemented to address different diffusion kinetics of the heterogeneous system for the in-vitro and in-vivo scenario (Fig. S1). For every compartment a set of diffusion coefficients and partition coefficients with respect to adjacent compartments was assigned. The underlying diffusion equation (Eq. 3) was solved numerically using an implicit Crank-Nicolson finite differences solver (32).

$$
\frac{\partial c(x, t)}{\partial t}=\nabla(D \nabla c(x, t))
$$

\section{Eq. 3}

Phase transitions were modelled in two steps. First the change of diffusivity at compartment boundaries was addressed by decomposing the Crank-Nicolson scheme into in- and outgoing fluxes and by a subsequent substitution of every diffusion coefficient in the Crank-Nicolson scheme for a given cell (position in the discretized spatial domain) with the harmonic mean of the diffusion coefficients of its adjacent cells according to work of Seta et al. (Eq.5 in (33)). Partitioning was incorporated into the Crank-Nicolson scheme by reducing back diffusion (multiplication of assigned diffusivity in a cell with partition coefficient $K$ ) for the direction of a "negative jump" $(K<1)$.

A sufficient spatial resolution at phase transitions is crucial to achieve stable and reasonable results in simulation but can largely slow down the speed of computation. To overcome this problem while gaining maximum speed of computations a nonuniform grid approach according to (34) was applied. Here, a base space step size of $1 \mu \mathrm{m}$ was used within every compartment. Towards phase transitions the step size was exponentially decreased to at least $1 / 10 \mu \mathrm{m}$. 
Dirichlet boundary conditions and reflecting boundary conditions were implemented to model infinite dosing and finite dosing, respectively.

At the start of the calculation, the concentration in the donor was set to the concentration of the drug in the ointment base as employed in the experiment. The concentration in all other elements was zero.

As basis for calculations the following assumptions were made: The thickness of the SC was assumed to be $15 \mu \mathrm{m}$ for in-vitro simulations and $17 \mu \mathrm{m}$ for in-vivo simulations that matches the findings from the tape-stripping procedure. The thickness of the deeper skin layers was assumed to be $2000 \mu \mathrm{m}$. The thickness of the donor was calculated from the amount of ointment base applied and the specific density of the ointment base $\left(=0.836 \mathrm{~g} / \mathrm{cm}^{3}\right)$. While the donor could be depleted of the drug, the tissue following the deeper skin layers was assumed to be a perfect sink (the concentration at the end of the deeper skin layers was always zero).

Due to the lack of blood level data no blood compartment was modelled.

\section{Model input parameters, fitting and prediction}

Determination of diffusivity in the ointment was performed as mentioned previously. Diffusivity in the deeper skin layers was computed as the harmonic mean of values taken from the literature $(24,25)\left(D_{D S L}=8166.67 \mu \mathrm{m}^{2} / \mathrm{h}\right)$. Partition coefficient $K_{S C / D S L}$ was taken from $(25)\left(K_{S C / D S L}=4.36\right)$.

$K_{S C \text { Nehicle }}$ (henceforth referred to as $K_{S C}$ ) and $D_{S C}$ were fitted using a non-linear least squares approach according to the following protocol:

1. The concentration-depth profiles for every time point were fitted separately (approach 1).

2. The total set of concentration-depth profiles for all times was fitted in one step (approach 2). 
This procedure was applied to infinite and finite dose data from FD-C and SB-M systems separately. For the in-vivo scenario, only approach 2 was applied.

After comparison of the findings, in a first step the kinetic parameters from the in-vitro infinite dose case were used to predict the in-vitro finite dose scenario - a procedure that could be successfully applied before with the help of complex 2D diffusion models for the simulation of drug release from an aqueous formulation $(22,25)$.

In a second step the potential to predict the in-vivo scenario from parameters fitted from the in-vitro case was examined.

\section{Software and programming}

The diffusion model was implemented in $\mathrm{C}++$ and compiled using the Apple LLVM version 5.0. Fitting was performed with the $\mathrm{R}$ programming language version 2.15.1 (35). For statistical evaluation GraphPad Prism version 6.0 was used.

\section{Results}

\section{Estimation of ointment diffusivity of FFA}

No significant differences could be found in the fitting results using Eq. 1 and Eq.2 (relative differences of $<0.01 \%$ ).

Distribution of fitting results of the diffusion coefficient of FFA in the ointment released over two different membrane systems (dialysis membrane and cellulose membrane) showed significant differences between the two groups (dialysis vs. cellulose membrane) with $p=0.02$ (Wilcoxon-Mann-Whitney-test). All single fits could be considered highly significant $(p<0.01)$. In comparison to results from the cellulose membrane, release over the dialysis membrane is accelerated by a factor of approximately 1.6 . 
Since variation of vehicle diffusivity in the given range did not alter concentrationdepth profiles of the SC significantly for various test simulations (e.g. maximum differences in concentration of $<0.01 \%$ for late times in the more error prone finite dose scenario), for the subsequent simulations the harmonic mean of all estimated diffusion coefficients with $D_{\text {vehicle }}=6583.19 \mu \mathrm{m}^{2} / \mathrm{h}$ was used as a model input parameter.

\section{Fitting of in-vitro infinite dose profiles}

Experimental results for both FD-C and SB-M showed a characteristic jump in concentration at the Vehicle/SC boundary given a vehicle initial concentration of 7.55 $\mathrm{mg} / \mathrm{ml}$ (Fig. 1). Here, concentrations in the upper segment of the SC are slightly higher for the SB-M setup in comparison to the FD-C. Both sets of experiments approach towards steady state, whereas SB-M experiments tend to reach steadystate slower. Average relative standard deviation of experimental data is slightly higher for the FD-C setup (45\%) in comparison to the SB-M (38\%).

Fitting of SC diffusivity and partition coefficient showed reasonable results with low residual standard errors (Tab. I). Fig. 1 shows results from fitting every data point of all times in total (All times, Tab. I). For both setups individual fittings show higher theoretical permeabilities $k_{p}\left(k_{\mathrm{p}}=\frac{\boldsymbol{U} \times \boldsymbol{K}}{i}\right)$ for early times in comparison to late times with a higher average permeability for the FD-C setup (factor of 2.33 Tab. I).

\section{Fitting of in-vitro finite dose profiles}


As for the infinite dose scenario, experimental results for FD-C and SB-M showed a characteristic jump in concentration at the Vehicle/SC boundary (Fig. 2). SB-M experiments tend to reach towards a pseudo steady-state for late times. Average relative standard deviation of experimental data is slightly higher for the SB-M setup (41\%) in comparison to the FD-C (36\%).

Fitting of SC diffusivity and partition coefficient for finite dose experiments using a FD-C and SB-M setup showed very reasonable results with low residual standard errors (Tab. II, fit not shown). Simulation results (fitted model) showed a decline of 11 $\%($ FD-C) and $17 \%$ (SB-M) after $6 \mathrm{~h}$ of simulations for the vehicle concentrations indicating the transient nature of the underlying process. High permeability values could be detected for single fittings of short incubation times for both FD-C and SB-M (Tab. II).

Fitting of SC diffusivity and partition coefficient showed reasonable results with low residual standard errors (Tab. II). For both in-vitro setups fittings for every time point separately show higher theoretical permeabilities for early times in comparison to late times with an higher average permeability for the SB-M setup (factor of 1.64 Tab. II). Overall, finite dose permeabilities showed similar values in comparison to infinite dose experiments. (All times, Tab. I, Tab. II).

\section{Prediction of in-vitro finite dose profiles from infinite dose input parameters}

Given this evidence, prediction of the in-vitro finite dose scenario from input values gathered from the infinite dose case was carried out and showed reasonable results (Fig. 2) with low relative absolute errors of the fit (0.63 for FD-C and 0.61 for SB-M). 
In-vivo experimental data showed a characteristic jump of concentration below the initial concentration of the vehicle for all time points (Fig. 3). Concentration-depth profiles for the infinite and finite dose tend to establish a (pseudo) steady-state after $180 \mathrm{~min}$. To compare the in-vivo situation with in-vitro findings the model was tested on this set of data. Fittings of SC diffusivity and partition coefficient could describe the fate of the substance excellently (Fig. 3, dashed lines). However, fitted diffusivities showed extremely high values in comparison to in-vitro studies leading to high theoretical permeability values (Tab. III A). Partition coefficients showed a reduction of factor 4-5 in comparison to in-vitro studies (Tab. I All times, Tab. II All times, Tab. III A).

Due to the steady-state-like curve shape of both finite and infinite dose in-vivo studies (Fig. 3, dashed lines) fitting of diffusivity can obviously lead to problems since the variation of $D_{S c}$ does not alter the curve shape when approaching steady-state. To overcome this problem $D_{s c}$ was set to a fixed value (taken from in-vitro finite dose SB-M fittings of all time points to account for an average best description of all time points) and only the partition coefficient was varied by the fitting routine. Results of this approach are depicted in Fig. 3 (solid lines). In comparison to the first approach the fitting error did only slightly increase (Tab. III B). Fitted partition coefficients showed only minor differences and hence, permeability values decreased by a factor of approximately 4-6 (Tab. III A vs. Tab. III B).

In-vivo permeability values (Tab. III B) showed a decrease of factor 1.2-2.7 in comparison to the in-vitro infinite dose case (Tab. I) and a decrease of factor 2.0-3.2 in comparison to the in-vitro finite dose fittings (Tab. II). The modelled finite dose scenario reveals a FFA vehicle depletion of only $5 \%$. 
In second step, model input parameters taken from the in-vitro infinite dose and finite dose case (All times, Tab. I, Tab. II) were used to predict the in-vivo scenario yielding reasonable average relative errors of the fit (Tab. IV).

For both application scenarios (finite and infinite dose) input values from the SB-M finite dose fittings led to the smallest fitting error (prediction results depicted in Fig. 6). Simulations (Fig. 4) lead to reasonable results, especially for short times and concentrations below stratum disjunctum (36).

\section{Discussion}

\section{Fitting results and differences in in-vitro and in-vivo setups}

Model fitting results for infinite and finite dose in-vitro studies are in very reasonable agreement with the experimental findings (Fig. 1). Variation in diffusivity and partition coefficient (All times) can be explained by intra-individual variability in determined parameters from different experiments (37). Average relative standard deviations of in-vitro and in-vivo concentrations over depth and time do show insignificant differences in variability $(32-42 \%$ for in-vivo and $36-45 \%$ for in-vitro). These observations are in reasonable agreement with findings from literature (37). As stated before, finite dose experiments show a more semi-infinite nature during the time of application due to the rather low donor depletion (11-17\%).

The observed decrease of in-vitro diffusion coefficients over time for finite and infinite scenarios is in reasonable agreement with in-vitro findings from Hansen et al. (24) and even with in-vivo findings of Herkenne et al. (38). The variation over time might be the result of the prolonged incubation time that can lead to higher data variability and subsequently makes fitting the curves more difficult. In addition, the resistance of 
the epidermis may have an impact on the curve shape after longer incubation times (13). In contrast to the addressed diffusivity, variability of the fitted partition coefficient over time did not show a clear trend for both in-vitro systems (Tabs. I, II).

Next, the suitability of the presented in-silico approach to describe the in-vivo situation was investigated. In-vivo fittings of diffusivity and partition coefficient showed lower partition coefficients with values below 1 and high diffusivity and subsequently high theoretical permeabilities. In comparison to experimental data an excellent correlation could be found (Fig. 3, dashed lines).

Due to the steady-state nature of the concentration-depth profiles after short times fitting results for $D_{S C}$ are prone to error. Therefore, SC diffusivity was set to constant values gathered from in-vitro studies. Fitting results were in good agreement with experimental data (Fig. 3, solid lines) for all time points. Partition coefficients were still lower in comparison to in-vitro experiments. Due to low partitioning, an even lower donor depletion was calculated, explaining the similar shapes of finite and infinite dose in-vivo experiments.

Speaking of theoretical permeabilities, lower calculated permeabilies for in-vivo experiments (Tab. III B) in comparison to SB-M fitting results (Tab. I B, II B, All times) could be observed,

However, student's t-test of SM-B in-vitro setup versus in-vivo setup did not indicate significant differences for the infinite dose $(p=0.76)$ and finite dose scenario $(p=0.06)$. The found differences in partition coefficient between in-vitro and in-vivo studies may be attributed to a potential contamination of skin slabs with subcutaneous fatty tissue during the process of excision of ex-vivo skin samples (solubility of FFA in triglycerides: $7 \%$; solubility of FFA in wool wax ointment: $0.9 \%$ ) (39). Therefore, it can be concluded that the presented in-silico model is applicable to in-vitro as well as in-vivo data. 
Prediction of the in-vitro finite dose scenario from model input parameters from the infinite dose case

In 2013 for aqueous solutions it could be shown that parameters obtained from the in-vitro infinite dose case are suitable parameters to predict the in-vitro finite dose scenario $(22,25)$. The investigations cited employ more complex two-dimensional diffusion models that need a larger set of input parameters (e.g. SC lipid diffusivities, corneocyte diffusivities, SC lipid/corneocyte partition coefficients) in comparison to the model presented. Therefore, the question arose, if these findings also hold true for semi-solid preparations using a less complex diffusion model. We could confirm the findings of the aforementioned works by applying a one-dimensional sparseparameter model for a semi-solid donor formulation. Excellent agreement with experimental data for the prediction of in-vitro finite dosing of the two model systems (FD-C and SB-M) was found (Fig. 2). This supports the hypothesis that in-vitro predictions of finite dosing might be possible using input parameters from infinite dose experiments.

Prediction of the in-vivo scenario from model input parameters from the in-vitro case Work on predictions of in-vivo permeation is still scarce. Pirot et al. could predict the in-vivo permeability of 4-cyanophenol for $60 \mathrm{~min}$ of application from values fitted from tape-stripping data obtained after $15 \mathrm{~min}$ (40). Variability of permeability of in-vivo fittings of FFA reported here is in reasonable agreement with results of Pirot and coworkers. Permeabilities for the in-vitro setups over time do show slightly larger variations (2-fold to 6 -fold between highest and lowest permeability of individual setups). Therefore, fitting of all data points from all measured times in total seems to be the most reasonable option to capture the whole kinetic process over time. 
Using a simple analytical solution of the diffusion equation, Herkenne et al. could show the potential of in-vitro tape-stripping data to predict the situation in man (41). Parameters from the in-vitro and in-vivo case were in reasonable agreement, but also some significant differences were observed. In contrast to the study presented here, only the infinite dose scenario was examined, vehicle diffusivity and resistance of the deeper skin layers were not taken into account and porcine skin was used for in-vitro studies.

In this study, predicted human in-vivo concentration-depth profiles from data obtained from in-vitro cases using human skin showed promising results (Fig. 4). Short times (15-30 min) could be described excellently but concentrations in superficial SC depths are considerably overpredicted for later times (60-180 min). Obviously, differences in partition coefficients between in-vitro and in-vivo experimental findings yield to the aforementioned discrepancies. Hence, possible alterations of solubility due to preparation of ex-vivo skin samples (e.g. contamination with subcutaneous fatty tissue) should be taken into account when gathering kinetic descriptors from invitro settings to describe the transdermal transport of substances in man.

\section{Mathematical diffusion model}

In contrast to analytical solutions of the diffusion equation, numerical approaches can be considered more versatile due to generalization (infinite vs. transient scenario), extendibility (e.g. protein binding, evaporation, blood flow) and complexity (e.g. microscopic models of the SC, multi compartment approaches). The interested reader is kindly referred to overview articles about analytical solutions and numerical approaches in the field of modelling skin transport $(13,42,43)$. However, one major drawback of numerical solutions is often the speed of computation. Due to the high level of optimization (implicit finite differences, non-uniform gridding) the presented 
model is capable to be incorporated in complex operations, like fitting routines, but still run in high spatial resolution on consumer hardware for reasonable computational times.

Considering the complex nature of the human skin and the underlying transport kinetics, one has to bear in mind the following facts:

- In this study the morphological structure of the SC was simplified as a pseudo-homogenous membrane. This obviously, does not reflect the microscopic structure of the SC. This limitation can be overcome by building more complex microscopic models of the SC (44) but this would increase the number of unknown parameters (23) as well as greatly increase computational complexity, making the model less generally applicable.

- Additional mechanisms, such as binding were not considered but might influence parameters determined from in-vitro parameters as well as the underlying kinetic assumptions $(43,45,46)$.

- In-vivo blood flow was not modelled since no blood level data was available. This might influence in-vitro in-vivo correlation (47).

Comparison of FD-C and SB-M to gather input parameters to predict in-vivo skin penetration

Comparison of the two in-vitro systems for the determination of model input parameters to predict the in-vivo scenario shows a tendency to prefer the SB-M setup that typically describes the in-vivo case in a more natural way due to lower hydration states in comparison to the FD-C setup. However, the differences in average relative errors (Tab. IV) are small. Fitting errors (residual standard errors) for 
the in-vitro setups indicate slightly better results for the SB-M (Tabs. I, II). This indicates that SB-M concentration-depth profiles could be described better with the applied mathematical model and this subsequently might influence the slightly better prediction results.

\section{Conclusion}

The application of an one-dimensional numerical in-silico model to simulate skin penetration turns out to be useful to describe in-vitro experiments for different systems (FD-C and SB-M), as well as in-vivo experiments for the infinite and finite dose scenario. Input parameters gathered from the in-vitro infinite dose case can be used to describe the in-vitro finite dose scenario for both model systems. Despite apparent differences in calculated partition coefficients between in-vivo and in-vitro studies, prediction of in-vivo scenarios from input parameters calculated from the invitro case seems to be a reasonable option. There is evidence to prefer the more invivo like SB-M as an ethical inoffensive model to predict the fate of a substance in man but certainly, a careful evaluation for a wide range of different xenobiotics is necessary and should be addressed in future works.

The application of in-silico models and the concepts presented in this study, will hopefully lead to an improved prediction of the in-vivo situation and a better understanding of the underlying physicochemical processes.

\section{References}

1. European Comission. Regulation (EC) No 1907/2006 of the European Parliament and of the Council concerning the Registration, Evaluation, Authorisation and Restriction of Chemicals (REACH); 2006. 
2. Hayden CGJ, Cross SE, Anderson C, Saunders NA, Roberts MS. Sunscreen Penetration of Human Skin and Related Keratinocyte Toxicity after Topical Application. Skin Pharmacol Phys. 2005;18(4):170-174.

3. Kim D, Andersen ME, Nylander-French LA. Dermal Absorption and Penetration of Jet Fuel Components in Humans. Toxicol Lett. 2006;165(1):1121.

4. European Commission. Council Directive on the Approximation of the Laws of the Member States Relating to Cosmetic Products (76/768/EEC). In.OJ L 262; 1976. p. $169-200$.

5. Rossignol MR. The 7th Amendment to the Cosmetics Directive. Atla-Altern Lab Anim. 2005;33(Suppl. 1):19-22.

6. European Comission. Review report for the active substance flufenacet. In: Directorate-General ECHCP, editor.; 2003.

7. European Comission. Review Report for the Active Substance Propineb. In: General ECHCPD-, editor.; 2003.

8. EFSA. EFSA Panel on Plant Protection Products and their Residues (PPR). In.Guidance on Dermal Absorption; 2012.

9. Lehman PA, Raney SG, Franz TJ. Percutaneous Absorption in Man: In Vitro-in vivo Correlation. Skin Pharmacol Phys. 2011;24(4):224-230.

10. Wagner H, Kostka KH, Lehr CM, Schaefer UF. Drug distribution in Human Skin Using Two Different In Vitro Test Systems: Comparison With In Vivo Data. Pharm Res. 2000;17(12):1475-1481.

11. Wagner H, Kostka KH, Lehr CM, Schaefer UF. Human Skin Penetration of Flufenamic Acid: In Vivo/In Vitro Correlation (Deeper Skin Layers) for Skin Samples From the Same Subject. J Invest Dermatol. 2002;118(3):540-544.

12. Franz TJ, Lehman PA, Franz SF, North-Root H, Demetrulias JL, Kelling CK, Moloney SJ, Gettings SD. Percutaneous Penetration of NNitrosodiethanolamine Through Human Skin (in Vitro): Comparison of Finite and Infinite Dose Applications from Cosmetic Vehicles. Fund Appl Toxicol. 1993;21(2):213-221.

13. Selzer D, Abdel-Mottaleb MMA, Hahn T, Schaefer UF, Neumann D. Finite and Infinite Dosing: Difficulties in Measurements, Evaluations and Predictions. Adv Drug Deliver Rev. 2013;65(2):278-294. 
14. Wagner H. Charakterisierung des Arzneistofftransportes in Humanhaut unter In-vitro und In-vivo Bedingungen Sowie unter Beruecksichtigung des Einflusses Zweier In-vitro Testsysteme. In.PhD Thesis. Saarbruecken, Germany: Saarland University; 2001. p. 235.

15. Melero A, Hahn T, Schaefer UF, Schneider M. In Vitro Human Skin Segmentation and Drug Concentration-skin Depth Profiles. In: Turksen K, editor. Permeability barrier: Humana Press; 2011. p. 33-50.

16. OECD. Guidance Document for the Conduct of Skin Absorption Studies. OECD Series on Testing and Assessment. Number 28. 2004.

17. OECD. Guideline for the Testing of Chemicals. Skin Absorption: In Vitro Method, 428. 2004.

18. European Commission. Basic Criteria for the In Vitro Assessement of Dermal Absorption of Cosmetic Ingredients. In: Products SCoC, editor.; 2006.

19. OECD. Guidance Notes on Dermal Absorption, 428. 2010.

20. World Health Organization. Dermal Absorption. In.Environmental Health Criteria 235; 2006. p. 90-103.

21. European Food Safety Authority. Guidance on Dermal Absorption. EFSA J. 2012;10(4):1-30.

22. Naegel A, Hahn T, Schaefer UF, Lehr CM, Heisig M, Wittum G. Finite Dose Skin Penetration: A Comparison of Concentration-depth Profiles from Experiment and Simulation. Comput Visual Sci. 2012;14(7):327-339.

23. Naegel A, Hansen S, Neumann D, Lehr CM, Schaefer UF, Wittum G, Heisig M. In-Silico Model of Skin Penetration Based on Experimentally Determined Input Parameters. Part li: Mathematical Modelling of In-Vitro Diffusion Experiments. Identification of Critical Input Parameters. Eur J Pharm Biopharm. 2008;68(2):368-379.

24. Hansen S, Henning A, Naegel A, Heisig M, Wittum G, Neumann D, Kostka $\mathrm{KH}$, Zbytovska J, Lehr CM, Schaefer UF. In-Silico Model of Skin Penetration Based on Experimentally Determined Input Parameters. Part I: Experimental Determination of Partition and Diffusion Coefficients. Eur J Pharm Biopharm. 2008;68(2):352-367.

25. Selzer D, Hahn T, Naegel A, Heisig M, Kostka KH, Lehr CM, Neumann D, Schaefer UF, Wittum G. Finite Dose Skin Mass Balance Including the Lateral 
Part: Comparison Between Experiment, Pharmacokinetic Modeling and Diffusion Models. J Control Release. 2013;165(2):119-128.

26. Borchert D. Methoden zur Untersuchung der Simultanen Penetration von Arzneistoffen und Vehikelbestandteilen aus Salben in Exzidierter Humanhaut. In.PhD Thesis. Saarbruecken: Saarland University; 1994.

27. Pershing LK, Corlett J, Jorgensen C. In Vivo Pharmacokinetics and Pharmacodynamics of Topical Ketoconazole and Miconazole in Human Stratum Corneum. Antimicrob Agents Chemother. 1994;38(1):90-95.

28. Theobald F. In-vitro Methoden zur Biopharmazeutischen Qualitaetspruefung von Dermatika unter Beruecksichtigung der Lipidzusammensetzung des Stratum Corneum. In.PhD Thesis. Saarbruecken: Saarland University; 1998.

29. Shah VP, Flynn GL, Yacobi A, Maibach HI, Bon C, Fleischer NM, Franz TJ, Kaplan SA, Kawamoto J, Lesko LJ, Marty JP, Pershing LK, Schaefer H, Sequeira JA, Shrivastava SP, Wilkin J, Williams RL. Bioequivalence of Topical Dermatological Dosage Forms - Methods of Evaluation of Bioequivalence. Pharm Res. 1998;15(2):167-171.

30. FDA. Guidance for Industry. Topical Dermatological Drug Product NDAs and ANDAs - In-vivo Bioavailability, Bioequivalance, In-vitro Release, and Associated Studie. In: Research CfDEa, editor.; 1998. p. 1-19.

31. Higuchi WI. Analysis of Data on the Medicament Release from Ointments. J Pharm Sci. 1962;51(8):802-804.

32. Crank J. In. The Mathematics of Diffusion. London: Oxford University Press; 1975. p. 49-53.

33. Seta Y, Ghanem AH, Higuchi WI, Borsadia S, Behl CR, Malick AW. Physical Model Approach to Understanding Finite Dose Transport and Uptake of Hydrocortisone in Hairless Guinea-pig Skin. Int J Pharm. 1992;81(1):89-99.

34. Babucke A, Kloker MJ, Rist U. Accuracy Analysis of the Fundamental Finitedifference Methods on Non-uniform Grids (Internal Report). In. Stuttgart Germany: Institut fuer Aerodynamik und Gasdynamik, Stuttgart University, Germany; 2009.

35. R Core Team. R: A Language and Environment for Statistical Computing. In. Vienna, Austria: R Foundation for Statistical Computing; 2012. 
36. Elias PM, Menon GK, Grayson S, Brown BE. Membrane Structural Alterations in Murine Stratum Corneum: Relationship to the Localization of Polar Lipids and Phospholipases. J Investig Dermatol. 1988;91(1):3-10.

37. Southwell D, Barry BW, Woodford R. Variations in Permeability of Human Skin Within and Between Specimens. Int J Pharm. 1984;18(3):299-309.

38. Herkenne C, Naik A, Kalia YN, Hadgraft J, Guy RH. Ibuprofen Transport Into and Through Skin from Topical Formulations: In Vitro-In Vivo Comparison. J Invest Dermatol. 2006;127(1):135-142.

39. Jaeckle E, Schaefer UF, Loth H. Comparison of Effects of Different Ointment Bases on the Penetration of Ketoprofen Through Heat-separated Human Epidermis and Artificial Lipid Barriers. J Pharm Sci. 2003;92(7):1396-1406.

40. Pirot F, Kalia YN, Stinchcomb AL, Keating G, Bunge A, Guy RH. Characterization of the Permeability Barrier of Human Skin In Vivo. Proc Natl Acad Sci USA. 1997;94(4):1562-1567.

41. Herkenne C, Naik A, Kalia YN, Hadgraft J, Guy RH. Dermatopharmacokinetic Prediction of Topical Drug Bioavailability In Vivo. J Invest Dermatol. 2006;127(4):887-894.

42. Naegel A, Heisig M, Wittum G. Detailed Modeling of Skin Penetration-An Overview. Adv Drug Deliver Rev. 2013;65(2):191-207.

43. Frasch HF, Barbero AM, Hettick JM, Nitsche JM. Tissue Binding Affects the Kinetics of Theophylline Diffusion Through the Stratum Corneum Barrier Layer of Skin. J Pharm Sci. 2011;100(7):2989-2995.

44. Hansen S, Naegel A, Heisig M, Wittum G, Neumann D, Kostka KH, Meiers P, Lehr CM, Schaefer UF. The Role of Corneocytes in Skin Transport Revised A Combined Computational and Experimental Approach. Pharm Res. 2009;26(6):1379-1397.

45. Hansen S, Selzer D, Schaefer UF, Kasting GB. An Extended Database of Keratin Binding. J Pharm Sci. 2011;100(5):1712-1726.

46. Anissimov YG, Roberts MS. Diffusion Modeling of Percutaneous Absorption Kinetics: 3. Variable Diffusion and Partition Coefficients, Consequences for Stratum Corneum Depth Profiles and Desorption Kinetics. J Pharm Sci. 2004;93(2):470-487.

47. Anissimov Y, Roberts M. Modelling Dermal Drug Distribution After Topical Application in Human. Pharm Res. 2011;28(9):2119-2129. 


\section{Tables}

Table I: $\quad$ Infinite dose fitting results $\left(D_{s c}, K_{s c}\right)$ of FFA concentration-depth profiles for FD-C experiments (A) and SB-M experiments (B) for different time points separately and total fit of all times points

\begin{tabular}{|c|c|c|c|c|c|}
\hline & Times $^{\mathrm{a}}$ & $D_{s c} \pm \mathrm{SE}^{\mathrm{c}}$ & $K_{s c} \pm \mathrm{SE}$ & $k_{p} \pm \mathrm{SE}^{\mathrm{d}}$ & $\mathrm{RSE}^{\mathrm{e}}$ \\
\hline \multirow[t]{5}{*}{ A } & 30 & $5.24 \pm 1.55$ & $1.93 \pm 0.29$ & $6.75 \mathrm{E}-01 \pm 2.24 \mathrm{E}-01$ & 0.63 \\
\hline & 60 & $5.71 \pm 2.66$ & $1.56 \pm 0.30$ & 5.99E-01 \pm 3.01E-01 & 1.07 \\
\hline & 180 & $6.36 \pm 1.46$ & $1.10 \pm 0.08$ & 4.67E-01 $\pm 1.13 \mathrm{E}-01$ & 0.51 \\
\hline & 360 & $1.84 \pm 0.47$ & $1.37 \pm 0.12$ & $1.68 \mathrm{E}-01 \pm 4.57 \mathrm{E}-02$ & 0.62 \\
\hline & All times ${ }^{b}$ & $6.53 \pm 1.98$ & $1.17 \pm 0.11$ & $5.09 \mathrm{E}-01 \pm 1.62 \mathrm{E}-01$ & 1.19 \\
\hline \multirow[t]{5}{*}{ B } & 30 & $3.67 \pm 2.59$ & $1.93 \pm 0.92$ & 4.74E-01 \pm 4.03E-01 & 1.25 \\
\hline & 60 & $2.23 \pm 1.48$ & $1.83 \pm 0.74$ & $2.71 \mathrm{E}-01 \pm 2.10 \mathrm{E}-01$ & 1.26 \\
\hline & 180 & $0.95 \pm 0.44$ & $2.24 \pm 0.60$ & 1.42E-01 \pm 7.63E-02 & 1.21 \\
\hline & 360 & $0.80 \pm 0.22$ & $1.97 \pm 0.25$ & $1.05 \mathrm{E}-01 \pm 3.21 \mathrm{E}-02$ & 0.77 \\
\hline & All times ${ }^{b}$ & $2.06 \pm 0.57$ & $1.59 \pm 0.20$ & 2.18E-01 $\pm 6.64 \mathrm{E}-02$ & 1.33 \\
\hline
\end{tabular}

a Units of min.

${ }^{\mathrm{b}}$ All time points $(30,60,180,360 \mathrm{~min})$ used for the fit.

${ }^{c}$ Units of $\mu \mathrm{m}^{2} / \mathrm{h}$.

${ }^{\mathrm{d}}$ Theoretical permeability $k_{p}$ calculated from $D_{s c}, K_{s c}$ and SC thickness. Units of $\mu \mathrm{m} / \mathrm{h}$.

${ }^{\mathrm{e}}$ Residual standard error of the fit.

Table II: $\quad$ Finite dose fitting results $\left(D_{s c}, K_{s c}\right)$ of FFA concentration-depth profilesfor FD-C experiments (A) and SB-M experiments (B) for different time points separately and total fit of all times points

\begin{tabular}{|c|c|c|c|c|c|}
\hline & Times $^{a}$ & $D_{s c} \pm \mathrm{SE}^{\mathrm{c}}$ & $K_{s c} \pm \mathrm{SE}$ & $k_{p} \pm \mathrm{SE}^{\mathrm{d}}$ & $\overline{\text { RSE }^{\mathrm{e}}}$ \\
\hline \multirow[t]{5}{*}{ A } & 30 & $9.65 \pm 1.96$ & $1.29 \pm 0.11$ & $8.28 \mathrm{E}-01 \pm 1.84 \mathrm{E}-01$ & 0.36 \\
\hline & 60 & $4.23 \pm 1.04$ & $1.05 \pm 0.11$ & 2.95E-01 \pm 7.95E-02 & 0.35 \\
\hline & 180 & $1.24 \pm 0.37$ & $2.25 \pm 0.35$ & $1.86 \mathrm{E}-01 \pm 6.18 \mathrm{E}-02$ & 0.82 \\
\hline & 360 & $1.36 \pm 0.14$ & $1.54 \pm 0.06$ & 1.39E-01 $\pm 1.56 \mathrm{E}-02$ & 0.25 \\
\hline & All times ${ }^{b}$ & $2.45 \pm 0.57$ & $1.58 \pm 0.17$ & $2.58 \mathrm{E}-01 \pm 6.61 \mathrm{E}-02$ & 1.07 \\
\hline \multirow[t]{5}{*}{ B } & 30 & $29.55 \pm 8.29$ & $0.71 \pm 0.07$ & $1.41 \mathrm{E}+00 \pm 4.17 \mathrm{E}-01$ & 0.36 \\
\hline & 60 & $8.55 \pm 6.29$ & $1.21 \pm 0.34$ & $6.91 \mathrm{E}-01 \pm 5.44 \mathrm{E}-01$ & 1.41 \\
\hline & 180 & $6.28 \pm 2.78$ & $0.88 \pm 0.13$ & 3.69E-01 $\pm 1.73 \mathrm{E}-01$ & 0.75 \\
\hline & 360 & $1.53 \pm 0.53$ & $1.41 \pm 0.18$ & $1.44 \mathrm{E}-01 \pm 5.28 \mathrm{E}-02$ & 0.77 \\
\hline & All times ${ }^{b}$ & $5.85 \pm 1.93$ & $1.08 \pm 0.12$ & 4.22E-01 $\pm 1.47 \mathrm{E}-01$ & 1.12 \\
\hline
\end{tabular}

a Units of min.

${ }^{b}$ All time points $(30,60,180,360 \mathrm{~min})$ used for the fit.

c Units of $\mu \mathrm{m}^{2} / \mathrm{h}$.

d Theoretical permeability $k_{p}$ calculated from $D_{S C}, K_{S C}$ and SC thickness. Units of $\mu \mathrm{m} / \mathrm{h}$.

e Residual standard error of the fit. 


\begin{tabular}{lllllll}
\hline & Experiment & $\boldsymbol{D}_{s c} \pm \mathbf{S E}^{\mathrm{a}}$ & & $\boldsymbol{K}_{s c} \pm \mathrm{SE}$ & $\boldsymbol{k}_{\boldsymbol{p}} \pm \mathbf{S E}^{\mathrm{b}}$ & $\mathbf{R S E}^{\mathrm{c}}$ \\
\hline $\mathbf{A}^{\mathrm{d}}$ & Infinite dose & 54.53 & \pm & $0.35 \pm 0.03$ & $1.12 \mathrm{E}+00 \pm 3.66 \mathrm{E}-01$ & 0.37 \\
& & 17.16 & & & & \\
& Finite dose & 36.60 & \pm & $0.26 \pm 0.02$ & $5.60 \mathrm{E}-01 \pm 1.59 \mathrm{E}-01$ & 0.24 \\
& & 10.04 & & & & \\
\hline $\mathbf{B}^{\mathrm{e}}$ & Infinite dose & $5.85 \pm 1.93$ & $0.55 \pm 0.06$ & $1.89 \mathrm{E}-01 \pm 6.58 \mathrm{E}-02$ & 0.62 \\
& Finite dose & $5.85 \pm 1.93$ & $0.38 \pm 0.04$ & $1.31 \mathrm{E}-01 \pm 4.53 \mathrm{E}-02$ & 0.37 \\
\hline
\end{tabular}

a Units of $\mu \mathrm{m}^{2} / \mathrm{h}$.

${ }^{\mathrm{b}}$ Theoretical permeability $k_{p}$ calculated from $D_{S C}, K_{s C}$ and SC thickness. Units of $\mu \mathrm{m} / \mathrm{h}$.

${ }^{c}$ Residual standard error of the fit.

${ }^{d}$ Fittings using all time points. Here $D_{s c}$ and $K_{s c}$ are objectives of the fitting procedure.

${ }^{e}$ Fittings using all time points. Here $D_{s c}$ were fixed and taken from SB-M finite dose fittings (Tab. II B, All times). Here only $\mathrm{K}_{\mathrm{sc}}$ was objective of the fitting procedure.

Table IV: Prediction errors of FFA concentration-depth profiles for in-vivo infinite dose and finite dose scenarios.

\begin{tabular}{lll}
\hline $\begin{array}{l}K_{s c} \text { and } D_{s c} \\
\text { taken from }\end{array}$ & $\begin{array}{l}\text { In-vivo infinite dose } \\
\text { prediction error }\end{array}$ & $\begin{array}{l}\text { In-vivo finite dose } \\
\text { prediction error }^{\mathrm{a}}\end{array}$ \\
\hline FD-C Infinite dose fittings & $9.18 \mathrm{E}-01$ & 1.21 \\
FD-C Finite dose fittings & $9.19 \mathrm{E}-01$ & 1.08 \\
\hline $\begin{array}{l}\text { SB-M Infinite dose } \\
\text { fittings }\end{array}$ & $9.15 \mathrm{E}-01$ & 1.06 \\
SB-M Finite dose fittings & $8.58 \mathrm{E}-01$ & 1.03 \\
\hline
\end{tabular}

${ }^{a}$ Errors are given as average relative errors. 

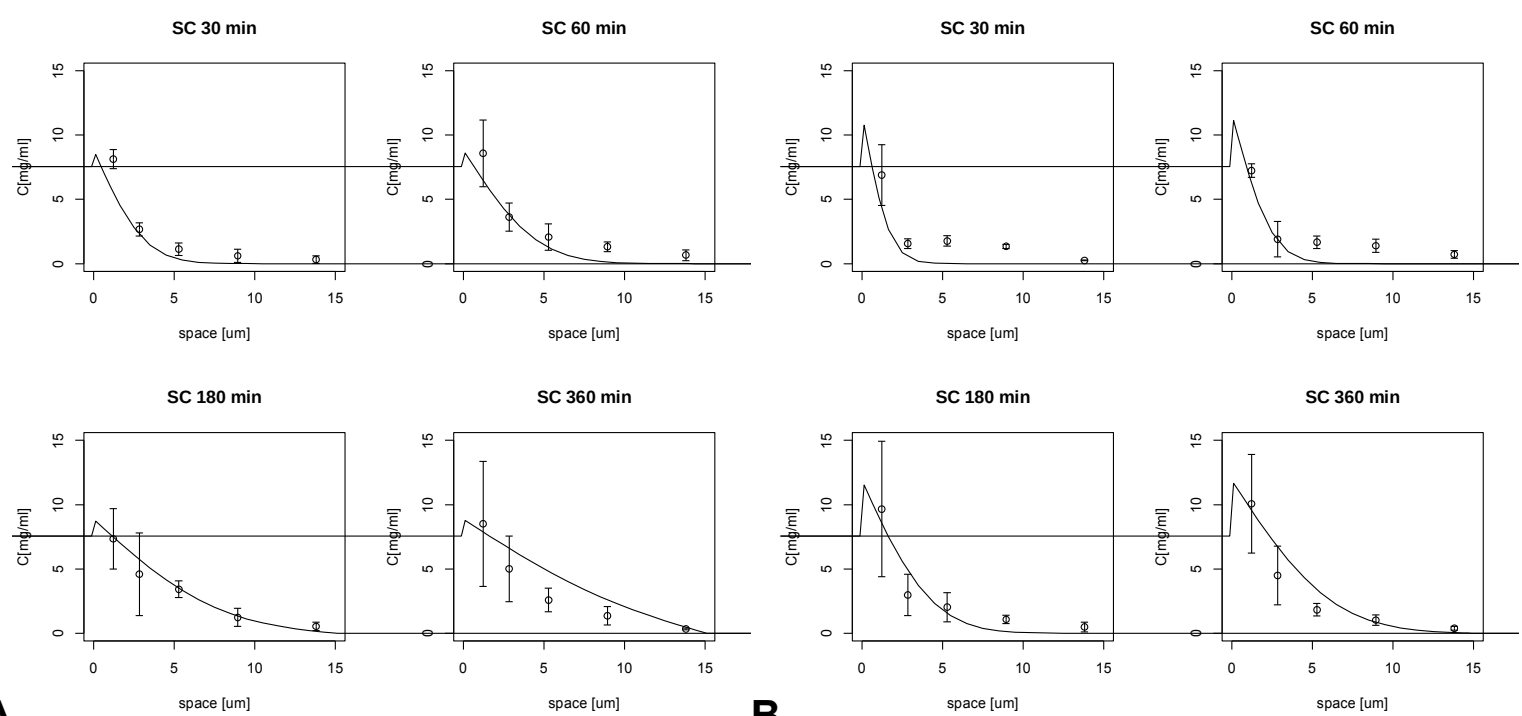

A

B

Figure 1: Overall fitted stratum corneum concentration-depth profiles of the infinite dose FD-C setup (A) and infinite dose SB-M setup (B) for various time points. Open circles denote experimental findings and solid lines denote the fitted model output. Data variation is given as standard deviation.
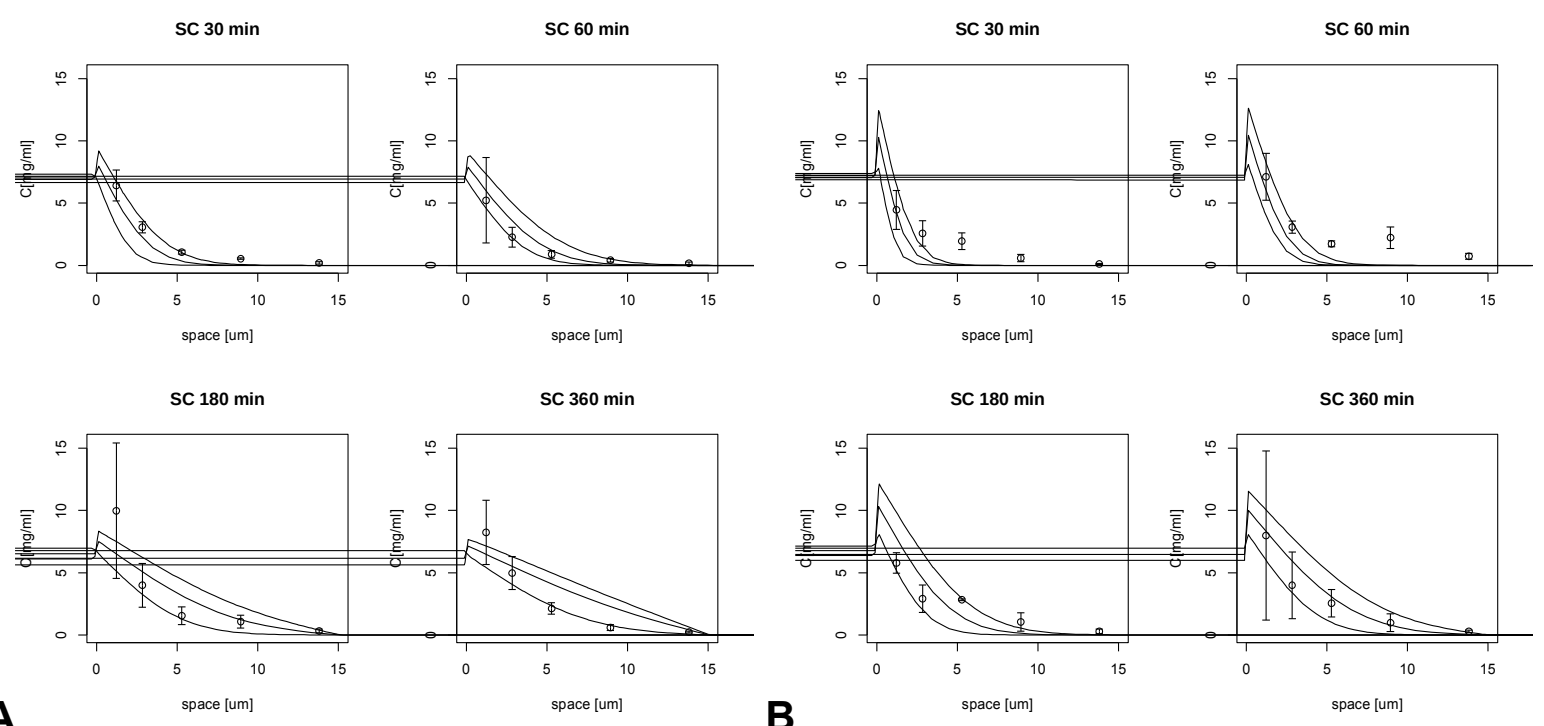

A

space [um] space [um] B

Figure 2: $\quad$ Predicted in-vitro finite dose experiments for the FD-C setup (A) and SB-M setup (B). Open circles denote experimental findings and solid lines denote the simulation results (prediction). Dashed lines indicate the $95 \%$ confidence interval. Data variation is given as standard deviation. 
$\mathrm{SC} 15 \mathrm{~min}$

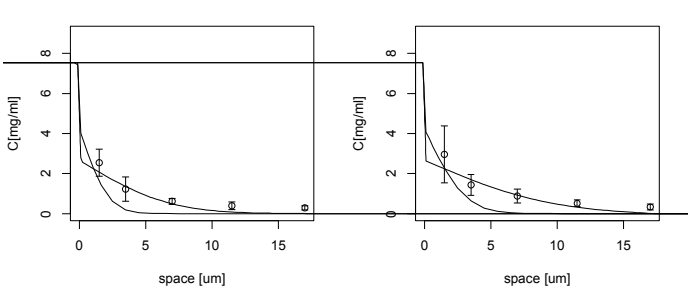

$\mathrm{SC} 60 \mathrm{~min}$

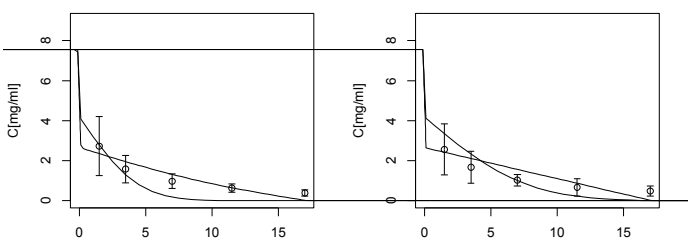

A

Figure 3: $\quad$ Fitted stratum corneum concentration-depth profiles of the invivo infinite dose case $(A)$ and finite dose case $(B)$ for various times with fixed DSC (solid line, values taken from in-vitro fittings) and free DSC (dashed line). Open circles denote experimental findings and solid lines denote the fitted model output. Data variation is given as standard deviation.
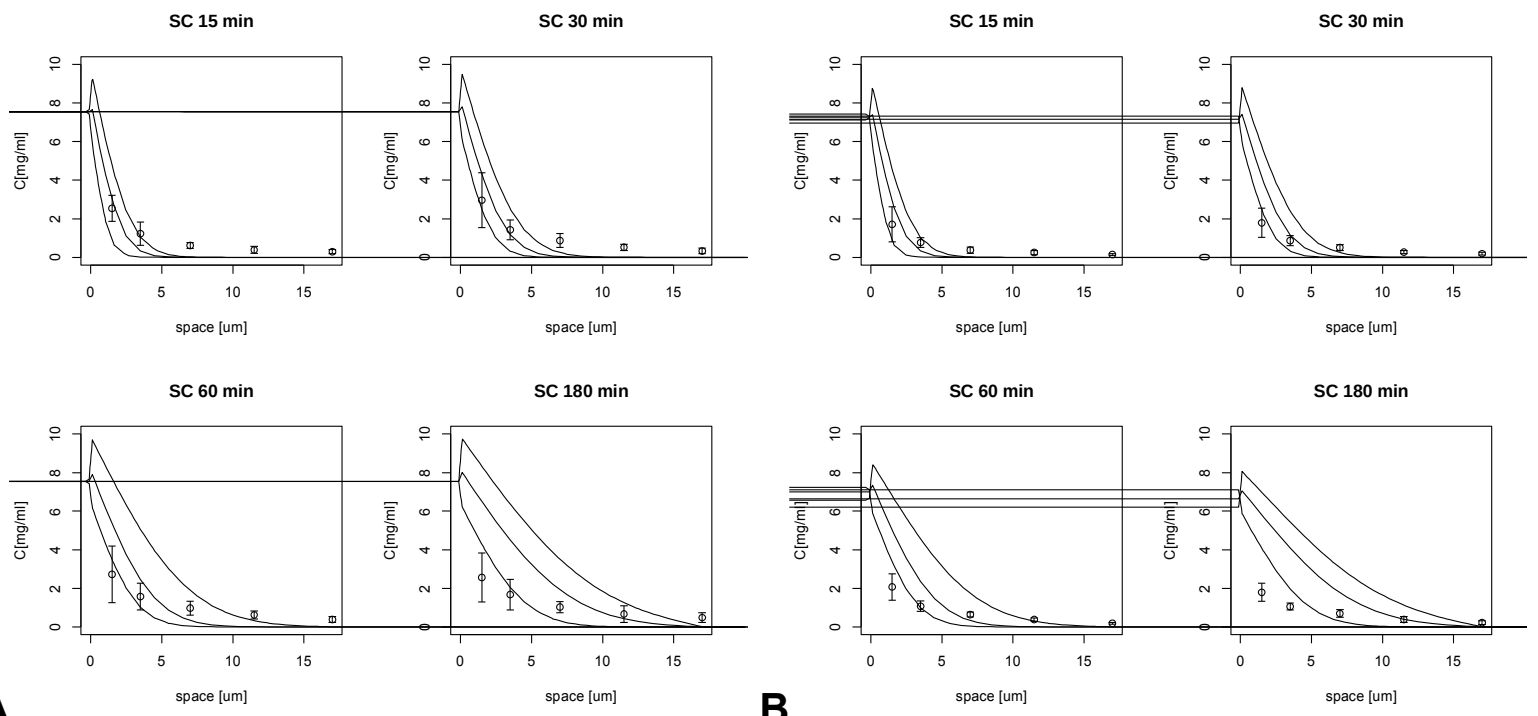

A

Figure 4:
Predicted in-vivo experiments for the infinite dose case $(A)$ and finite dose case (B). Open circles denote experimental findings and solid lines denote the simulation results (prediction). Dashed lines indicate the $95 \%$ confidence interval. Data variation is given tas standard deviation. 